\title{
Mechanism of Secondary Deformation of Extruded AZ31 Magnesium Alloy by Viscoplastic Self-Consistent Model
}

\author{
Su Hui, ${ }^{1}$ Zhibing Chu $\mathbb{D},{ }^{1,2}$ Huanzhu Wang, ${ }^{1}$ Yugui Li $\mathbb{D},{ }^{1}$ Lifeng Ma, ${ }^{1}$ and Chun Xue ${ }^{1}$ \\ ${ }^{1}$ Engineering Research Center Heavy Machinery Ministry of Education, Taiyuan University of Science and Technology, \\ Taiyuan 030024, China \\ ${ }^{2}$ School of Mechanics and Architectural Engineering, Jinan University, Guangzhou, 510632, China \\ Correspondence should be addressed to Zhibing Chu; chuzhibing@tyust.edu.cn
}

Received 11 December 2019; Revised 2 March 2020; Accepted 17 March 2020; Published 15 May 2020

Academic Editor: Gianfranco Palumbo

Copyright (c) 2020 Su Hui et al. This is an open access article distributed under the Creative Commons Attribution License, which permits unrestricted use, distribution, and reproduction in any medium, provided the original work is properly cited.

\begin{abstract}
The viscoplastic self-consistent (VPSC) model is used to establish a combination of different deformation mechanisms. By using this model, axial tension and compression tests of extruded AZ31 magnesium alloy at room temperature are simulated. The influence of secondary deformation mechanism (prismatic $<a>$ slip, pyramidal $<c+a>$ slip, and $\{10 \overline{1} 1\}$ compression twin) on mechanical response and texture evolution is expounded. Increased activity of the prismatic $<a>$ slip is conducive for the improvement of flow stress in mechanical response during axial tension and for the splitting of pole densities in the $\{0002\}$ pole figure during axial compression. However, increased activity of the pyramidal $\langle c+a\rangle$ slip causes the basal texture to transfer to the extrusion direction in the $\{0002\}$ pole figure during axial compression. The $\{10 \overline{1} 1\}$ compression twinning has a negligible influence on the plastic deformation and mechanical response of AZ31 magnesium alloy during axial tension and compression. However, the $\{10 \overline{1} 1\}$ compression twinning should be included in VPSC modeling to predict the texture evolution accurately.
\end{abstract}

\section{Introduction}

Magnesium alloy has low density, high specific strength, and stiffness and is easy to recycle with good thermal conductivity. These characteristics enable the alloy to hold many application prospects in aerospace, communication, and other fields $[1,2]$. Among the magnesium alloys, AZ31 is one of the most widely applied variants. However, slip and twin are required to coordinate plastic deformation, due to the hexagonal closely packed structure of magnesium alloy, the low symmetry of the crystal materials, and the lack of independent slip systems, thereby adding complexity to the study of the plastic deformation mechanism of magnesium alloys [3]. Therefore, this study focuses on the slip and twin mechanisms in the plastic deformation of magnesium alloys to elucidate the reasonable and effective use of the plastic deformation mechanism. This work can provide technical support for the research and development of high-performance magnesium alloys (i.e., in terms of plasticity and strength).
Researchers have found that the deformation mechanism of magnesium alloys during plastic deformation mainly includes the following: $\{0001\}<11 \overline{2} 0\rangle$ basal $\langle a\rangle$ slip, $\{1010\}<11 \overline{2} 0>$ prismatic $<a>$ slip, $\{11 \overline{2} 2\}<\overline{11} 23>$ pyramidal $\langle c+>$ slip, $\{10 \overline{1} 2\}$ tension twin, and $\{10 \overline{1} 1\}$ compression twin [4]. The lower critical resolved shear stress (CRSS) of the basal $\langle a\rangle$ slip and $\{10 \overline{1} 2\}$ extension twin than that of other deformation mechanisms has been proven to be the main deformation mechanism that accommodates the plastic strain along and is perpendicular to the $c$-axis of crystal at room temperature [5]. Prismatic $<a>$ slip, pyramidal $\langle c+a\rangle$ slip, and $\{10 \overline{1} 1\}$ compression twin also play important roles in the plastic deformation of AZ31 magnesium alloy at room temperature [6]. In the present paper, the three deformation mechanisms are called secondary deformation mechanisms on the basis of their contributions to plastic deformation. However, studies on the contribution of these secondary deformation mechanisms during plastic deformation are few. 
To date, researchers on the plastic deformation mechanism of metal materials have developed several models. The viscoplastic self-consistent (VPSC) model is the most representative model proposed by Lebensohn and Tome [7]. This model introduces the rate-dependent constitutive relation of Asaro and Needlema [8] and considers the slip, twinning, and anisotropy of the material. Moreover, the stress and strain coordination at the grain boundary is satisfied, and the large deformation behavior of polycrystalline materials can be simulated and predicted. At present, the VPSC model has been successfully used to simulate the macroscopic mechanical response and microtexture evolution of magnesium alloys [5]. The method adopted in this study is to change the deformation mechanism embedded in the VPSC model systematically. The influence of secondary deformation mechanism on mechanical response and the microtexture evolution of magnesium alloys during plastic deformation is studied. This aspect is significant for the expansion of the application of magnesium alloys at room temperature.

\section{VPSC Model}

The VPSC model considers the interaction between grains in polycrystals and assumes that the grains are ellipsoid. It is included and buried deep in a homogeneous equivalent medium, and the model adopts a rate-dependent continuous constitutive model equation [9]:

$$
\begin{aligned}
\varepsilon_{i j}(\bar{X}) & =\sum_{s} m_{i j}^{s} \gamma^{s}(\bar{X})=\gamma_{0} \sum_{s} m_{i j}^{s}\left\{\frac{m_{k l}^{s} \sigma_{k l}(\bar{X})}{\tau^{s}}\right\}^{n} \\
& =M_{i j k l} \sigma_{k l}(\bar{X})
\end{aligned}
$$

where $\tau^{s}$ denotes the critical shear stress, $m_{i j}^{s}=1 / 2\left(n_{i}^{s} b_{j}^{s}+\right.$ $\left.b_{j}^{s} n_{i}^{s}\right)$ is the Schmid factor of the slip system/twin system $(s)$, and $n^{s}$ and $b^{s}$ pertain to the normal direction (ND) of the slip/twin plane and the slip/twin direction, respectively. $\varepsilon_{i j}(\bar{x})$ and $\sigma_{k l}(\bar{x})$ are strain and stress partial tensors, respectively. $\gamma^{s}$ stands for the local shear rate acting on the slip system $(s) \cdot \gamma_{0}$ represents the normalized coefficient, and $n$ is the rate sensitivity index. $M_{i j k l}$ stands for the viscoplastic convention and can relate the macroscopic strain rate to the macroscopic deviatoric stress.

2.1. Voce Hardening Model. The critical shear stress $\tau^{s}$ varies with plastic deformation, and the evolution rules can be described by the Voce hardening model:

$$
\tau^{s}=\tau_{0}^{s}+\left(\tau_{1}^{s}+\theta_{1}^{s} \Gamma\right)\left(1-\exp \left(-\frac{\theta_{0}^{s} \Gamma}{\tau_{1}^{s}}\right)\right),
$$

where $\Gamma=\sum_{s} \Delta \gamma^{s}$ is the cumulative shear of the grain, $\tau_{0}$ denotes the initial critical stress, $\theta_{0}$ stands for the initial hardening rate, $\theta_{1}$ refers to the saturation hardening rate, and $\tau_{0}^{s}$ and $\tau_{1}^{s}$ are the initial and extrapolated critical shear stresses of mechanism $s$, respectively. Figure 1 depicts the specific definitions.

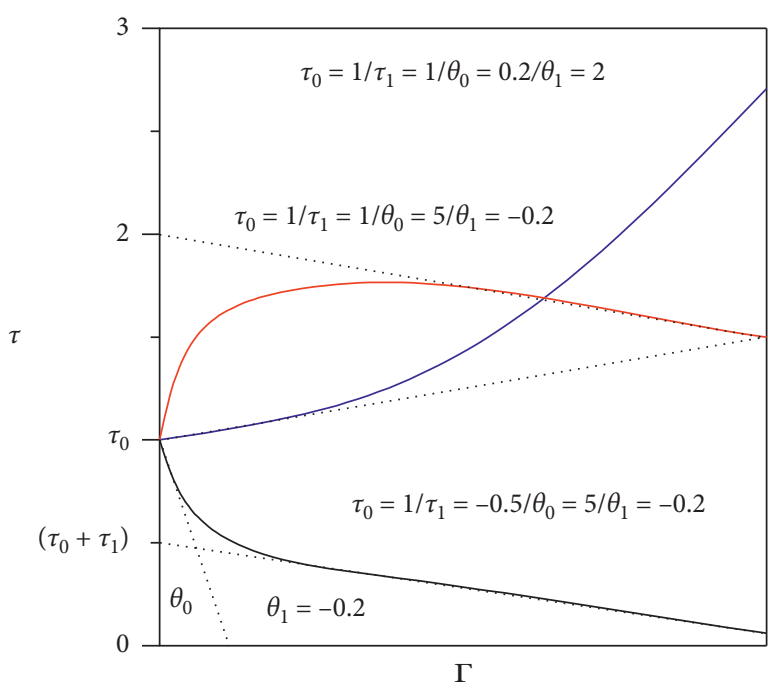

Figure 1: Meaning of parameters in voce hardening.

Slip hardening coefficient $h^{s s^{\prime}}$ is introduced to consider the interaction among different deformation modes. Therefore, the increment of critical shear stress can be expressed as follows:

$$
\Delta \tau^{s}=\frac{\mathrm{d} \widehat{\tau}^{s}}{\mathrm{~d} \Gamma} \sum_{s^{\prime}} h^{s s^{\prime}} \Delta \gamma s^{\prime},
$$

where $\Delta \gamma s^{\prime}$ is the shear increment and $\mathrm{d} \widehat{\tau}^{s} / \mathrm{d} \Gamma=\left[\theta_{1}+\left(\left(\mid \theta_{0}\right.\right.\right.$ $\left.\left./ \tau_{1} \mid\right) \tau_{1}-\theta_{0}\right) \exp \left(-\Gamma\left(\left|\theta_{0} / \tau_{1}\right|\right)\right)+\left(\left|\theta_{0} / \tau_{1}\right|\right) \theta_{1} \Gamma \exp \left(-\Gamma\left(\mid \theta_{0}\right.\right.$ $\left.\left.\left./ \tau_{1} \mid\right)\right)\right]$

2.2. Predominant Twinning Reorientation (PTR). VPSC considers the slip and twin plastic deformation mechanisms [10]. In this study, PTR [11] is used to analyze the effect of twins on texture evolution during plastic deformation. For grain $g, \gamma^{t, g}$ is the shear strain caused by each twin $t$, and the corresponding twin volume fraction is $V^{t, g}=\left(\gamma^{t, g} / S^{t}\right)$, where $S^{t}$ is the intrinsic shear strain of twin system $t$.

The integral number of twins caused by all twin systems in all grains is called the cumulative twin integral number:

$$
V^{\text {acc,mode }}=\sum_{g} \frac{\sum_{t} \gamma^{t, g}}{S^{t}}
$$

By superimposing the twin fraction of each incremental step, the cumulative twin fraction is compared with the critical volume fraction caused by twins. Thus, the critical volume fraction is defined as follows:

$$
V^{\text {th,mode }}=A^{\text {th1 }}+A^{\text {th } 2} \frac{V^{\text {eff,mode }}}{V^{\text {acc,mode }}},
$$

where $V^{\text {eff,mode }}$ is the effective twin crystal integral number and $A^{\text {thl }}$ and $A^{\text {thl }}$ are material constants.

\section{Experimental Methods}

The initial material selected in this study was a commercial extruded AZ31 magnesium alloy bar with a diameter of 
$40 \mathrm{~mm}$. The tension and compression experiments were conducted in the extrusion direction (ED). Among them, the tensile sample was the "dumbbell" shape with a total length of $36 \mathrm{~mm}$, a gauge length of $15 \mathrm{~mm}$, and a cross-section size of $5 \mathrm{~mm} \times 1 \mathrm{~mm}$, as shown in Figure 2(a). The compressed sample was a cuboid of $8 \mathrm{~mm} \times 8 \mathrm{~mm} \times 12 \mathrm{~mm}$ with a loading direction of $12 \mathrm{~mm}$, as shown in Figure 2(b). The extrusion direction of magnesium alloy is expressed by ED, and the transverse and normal directions perpendicular to the extrusion direction are expressed by TD and ND, respectively. The inverse pole diagram of the three faces of the magnesium alloy was measured by EBSD technology and combined into a three-dimensional microstructure, as shown in Figure 3(a). As can be seen from the figure, most of the initial samples are equiaxed crystal structure, and the average grain size is $38 \mu \mathrm{m}$. Figure 3(b) shows the initial texture. As we can see, most of the c-axis of the grain was perpendicular to the ED; that is, the basal texture was parallel to the ED, which results in a strong basal texture in the initial pole figure distribution of magnesium alloy.

The MTEX toolbox of MATLAB was used to estimate the orientation distribution function (ODF) from the EBSD data by using the least squares method. In MTEX, the grain number was utilized as the control variable, and the ODF was discretized to output the grain orientation data of 2,000 grain numbers as the initial grain orientation of the VPSC model. The plastic deformation mechanism in the VPSC model mainly included the basal $\langle a\rangle$ slip, prismatic $\langle a\rangle$ slip, pyramidal $<c+a>$ slip, $\{10 \overline{1} 2\}$ tension twin, and $\{10 \overline{1} 1\}$ compression twin. It is assumed that the reference shear strain rate $\dot{\gamma}_{0}=0.001 \mathrm{~s}^{-1}$ and rate sensitivity index $m=0.05$ were used for all slip and twin systems. The elastic constants of a single-crystal magnesium at room temperature were [12] $C_{11}=58.0 \mathrm{GPa}, \quad C_{12}=25.0 \mathrm{GPa}, \quad C_{13}=20.8 \mathrm{GPa}, \quad C_{33}=$ $61.2 \mathrm{GPa}$, and $C_{44}=16.6 \mathrm{GPa}$. During simulation calculation, to satisfy the ellipticity of the sample, the strain rate in the loading direction was $D_{11}=0.001 \mathrm{~s}^{-1}$, and other macroscopic stress components were set to 0. Studies [13] have shown that, in the simulation of magnesium alloys, the simulation results of the affine model are the closest to the actual deformation of the material. Therefore, we used the affine model in this model. As suggested in the VPSC manual [14], we selected the rate sensitivity factor $n=20$ to simulate all rate-insensitive deformations at room temperature and twin deformation process.

Combined with the Voce hardening model and PTR twin model, the VPSC model was used to simulate and predict the true stress-strain curve of the AZ31 magnesium alloy under axial tension and compression, as shown in Figure 4. Notably, the AZ31 magnesium alloy had serious axial tension and compression asymmetry, which are mainly manifested in two aspects: (1) axial tension and compression yield asymmetry. The axial tensile yield stress $(144 \mathrm{MPa})$ was significantly higher than the axial compression stress (100 MPa), and the yield ratio was approximately 1.4. (2) The strain hardening rate was asymmetrical. During axial compression, the strain hardening rate changed significantly, and the stress-strain curve was similar to an "s" shape. During axial tension, the strain hardening rate underwent less changes, and the stress-strain curve gradually changed. The stress-strain curves predicted by the VPSC model were in good agreement with the experimental results and accurately reflected the stress-strain characteristics of each stage in the axial tension and compression plastic deformation process.

The optimal hardening parameters of the material were obtained by fitting the stress-strain curve with the VPSC model, as shown in Table 1. It is worth pointing out that, using this set of hardening parameters, we have obtained similar stress-strain curves as shown in Figure 4, indicating that the stress-strain curves of axial tension and compression obtained by this set of parameters are in good agreement with the experimental results. Therefore, we consider that the results of the discussion on the initiation of slip system and twin system based on this set of hardening parameters are acceptable. Results showed that, at room temperature, the CRSS of basal $<a>$ slip [15] (approximately $0.45-0.81 \mathrm{MPa})<$ the CRSS of the $\{10 \overline{1} 2\}$ tension twin [16] (approximately $2-2.8 \mathrm{MPa}$ ) $<$ the CRSS of the prismatic $<a>$ slip [17] (approximately $39.2 \mathrm{MPa}$ ) $<$ the CRSS of the pyramidal $<c+a>$ slip [5] (approximately $45-81 \mathrm{MPa})<$ the CRSS of the $\{1011\}$ compression twin [16] (approximately $76-153 \mathrm{MPa}$ ) of magnesium and its alloys. Table 1 presents that the CRSS of each simulated deformation mechanism was relatively high, but the overall size order was consistent with the research value. Notably, the CRSS values of each deformation mechanism were simultaneously affected by grain size, grain shape, solid solution, defect structure, and other factors. Therefore, the CRSS values used by different scholars in the VPSC simulation process were different. For example, Ma [18] obtained that the CRSS value of the basal slip was $28 \mathrm{MPa}$ at room temperature, and the CRSS of tensile twin was $65 \mathrm{MPa}$, whereas Agnew [19] obtained a CRSS value of tension twin at $30 \mathrm{MPa}$. In the present study, the optimal hardening parameters of the deformation mechanisms of magnesium alloys were obtained by fitting the experimental stress-strain curves, which will play an important guiding role for future researchers to study the macro- and microdeformation mechanisms of magnesium alloys.

Many scholars $[6,20]$ have verified that the basal $<a>$ slip and $\{1012\}$ tension twins have relatively low CRSS values, such that the main deformation mechanisms in the plastic deformation process of magnesium alloys occur at room temperature. Therefore, the present study focused on the numerical investigation of the effects of the secondary deformation mechanism with high CRSS values in the plastic deformation process of magnesium alloy. The VPSC models of the four combinations of deformation mechanisms were constructed, as shown in Table 2. The deformation mechanism of combination A included basal $\langle a\rangle$ slip, prismatic $<a>$ slip, pyramidal $\langle c+a\rangle$ slip, $\{10 \overline{1} 2\}$ tension twin, and $\{10 \overline{1} 1\}$ compression twin. For combination $B$, basal $<a>$ slip, pyramidal $\langle c+a\rangle$ slip, $\{10 \overline{1} 2\}$ tension twin, and $\{10 \overline{1} 1\}$ compression twin constituted the deformation mechanism. Combination $C$ included basal $\langle a\rangle$ slip, prismatic $\langle a\rangle$ slip, $\{10 \overline{1} 2\}$ tension twin, and $\{10 \overline{1} 1\}$ compression twin, whereas combination $D$ consisted of basal $\langle a\rangle$ slip, prismatic $\langle a\rangle$ slip, pyramidal $<c+a>$ slip, and $\{10 \overline{1} 2\}$ tension twin. 


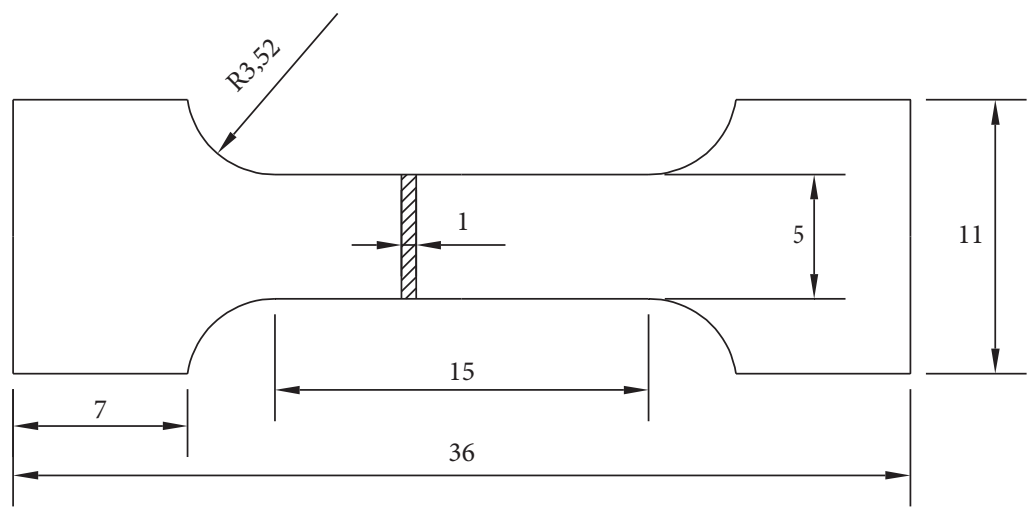

(a)

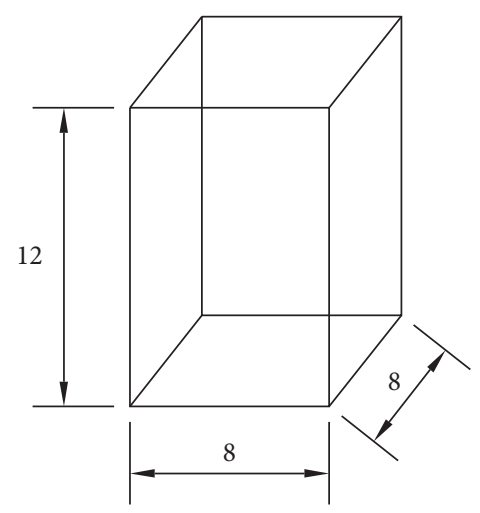

(b)

Figure 2: Sample shape and size: (a) tensile sample; (b) compression sample.

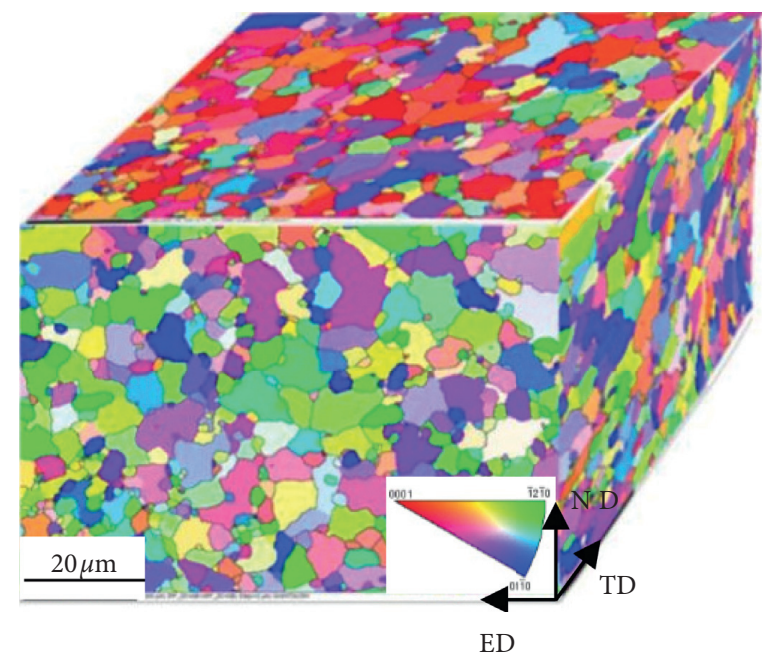

(a)
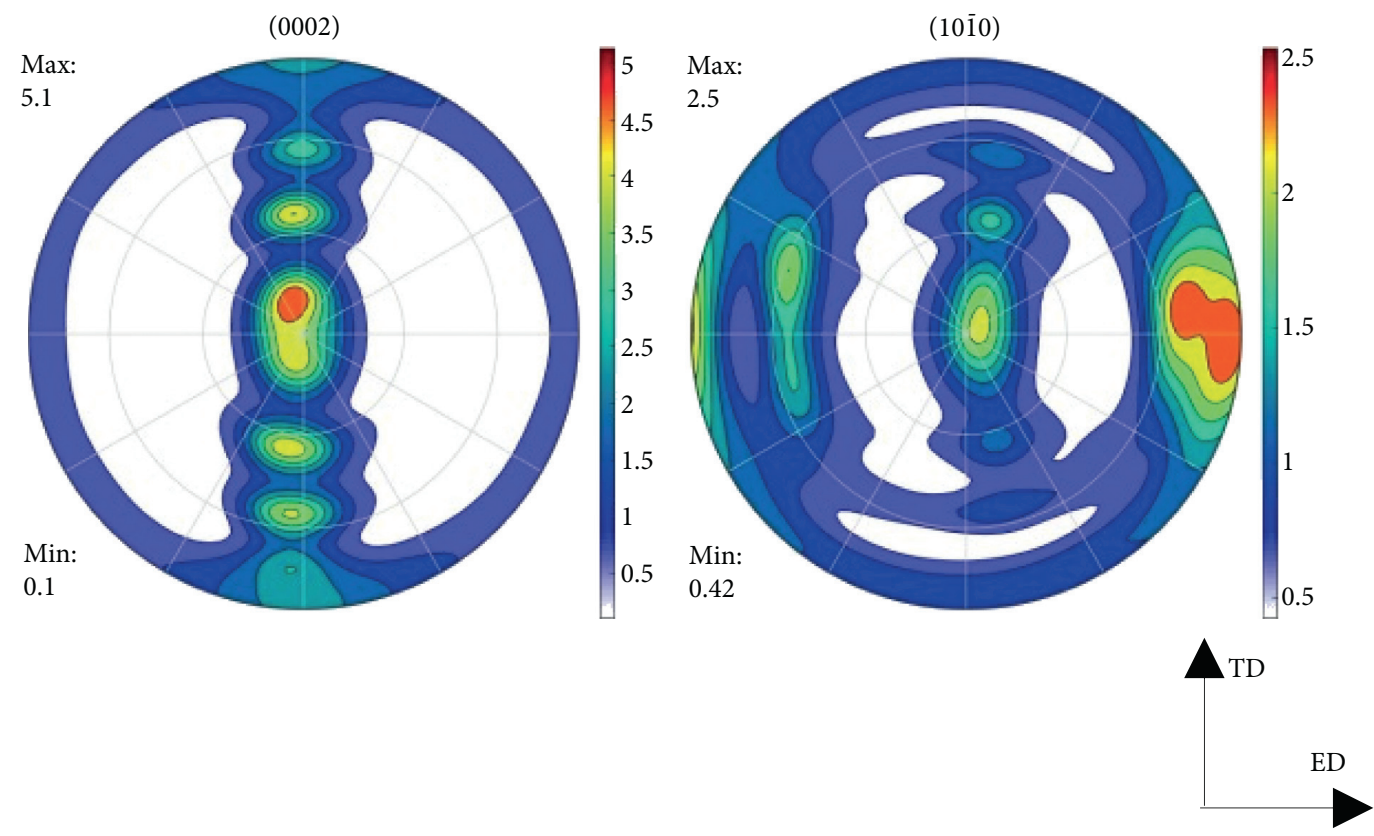

(b)

Figure 3: Initial state of MgAZ31 extruded bar: (a) three-dimensional microstructure; (b) initial texture. 


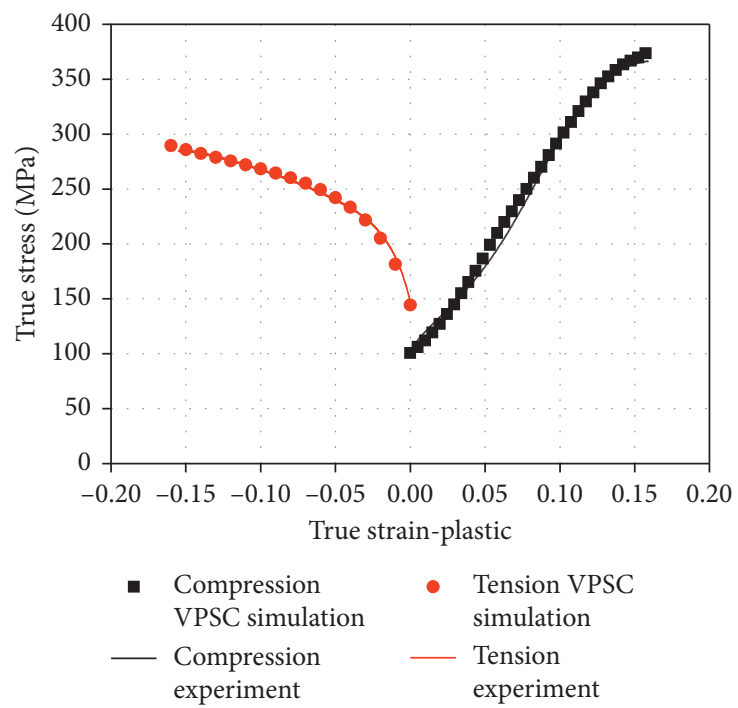

FIGURE 4: Correlation between experimental stress-strain and VPSC simulation results at room temperature.

TABLE 1: Hardening parameters of slip and twin systems in the simulation of Mg alloy under monotonic loading.

\begin{tabular}{lcccccc}
\hline Deformation mode & $\tau_{0}(\mathrm{MPa})$ & $\tau_{1}(\mathrm{MPa})$ & $\theta_{0}(\mathrm{MPa})$ & $\theta_{1}(\mathrm{MPa})$ & $\dot{\gamma}_{0}$ & $A^{\text {th1 }}$ \\
\hline Basal $\langle a\rangle$ & 28 & 60 & 185 & 16 & 0.001 & \\
Prismatic $\langle a\rangle$ & 85 & 10 & 200 & 250 & 0.001 & \\
Pyramidal $\langle c+a\rangle$ & 110 & 15 & 120 & 400 & 0.001 & \\
Extension twin & 45 & 0 & 150 & 400 & 0.001 & 0.1 \\
Compression twin & 210 & 100 & 345 & 400 & 0.001 & 0.1 \\
\hline
\end{tabular}

TABLE 2: VPSC model with different deformation mechanisms.

\begin{tabular}{lll}
\hline Designation & Basal $\langle a\rangle$ \\
Combination A & Prismatic $\langle a\rangle$ \\
Combination B & Pyramidal $\langle c+a\rangle$
\end{tabular}

\section{Results and Discussion}

Figure 5 shows the predicted stress-strain curves in the axial compression process and corresponding slip and twinning activities in the different combinations of deformation mechanisms. Figure 5(a) presents a minimal discrepancy between the stress-strain curve of combinations $A$ and $D$. On the contrary, the stress-strain curve of combinations $B$ and $C$ changed significantly. Flow stress and yield limit were significantly higher than those of combination $A$. Figure 5(b) exhibits that the activity of tension twins increased up to $54 \%$ in the initial deformation stage due to the low CRSS values at room temperature. Moreover, the c-axis of the grain was subjected to tension stress during axial compression, which is conducive for the activity of the tension twins. When the prismatic $\langle a\rangle$ slip was eliminated from the adopted 

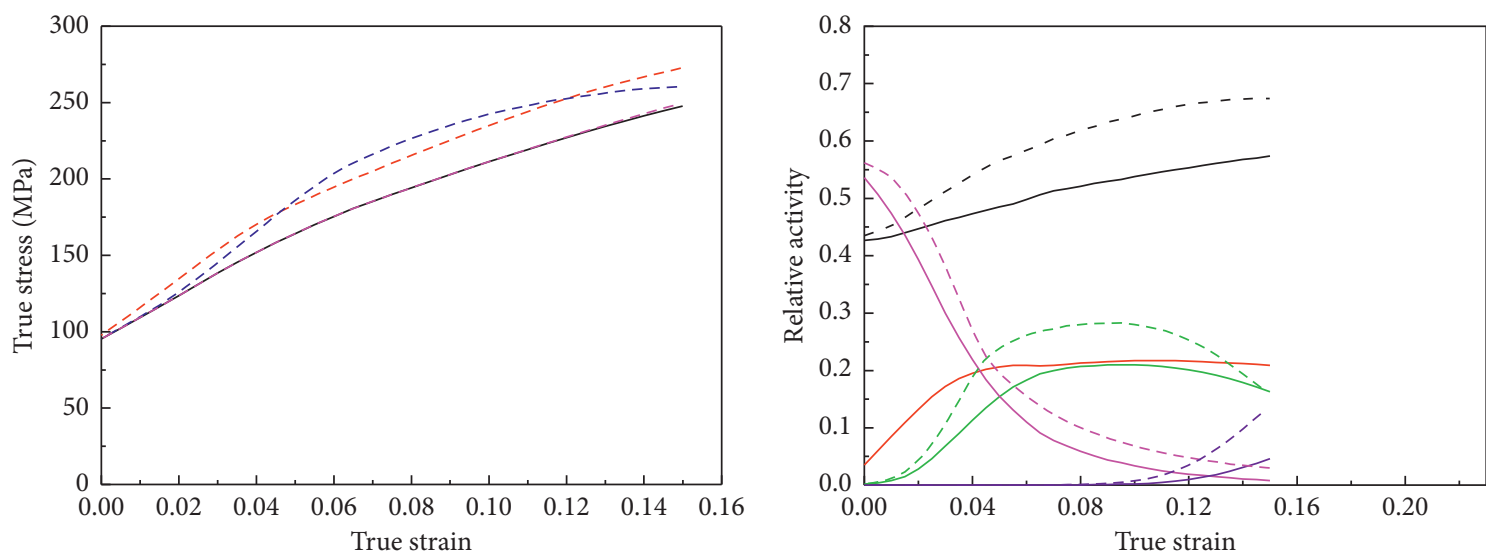

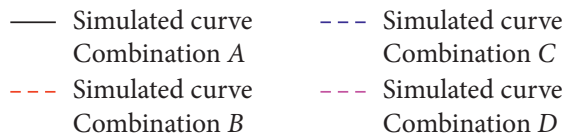

(a)

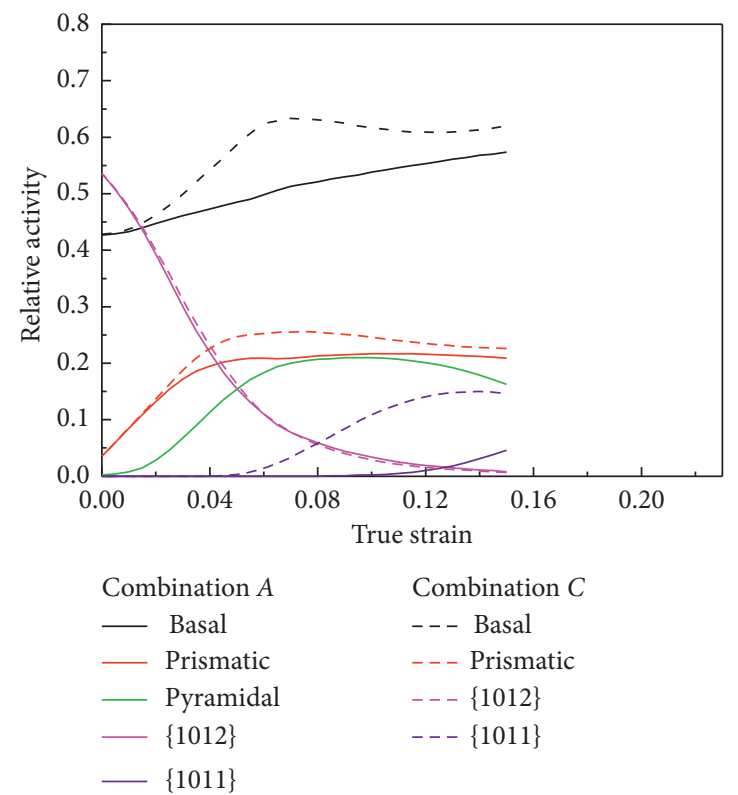

(c)

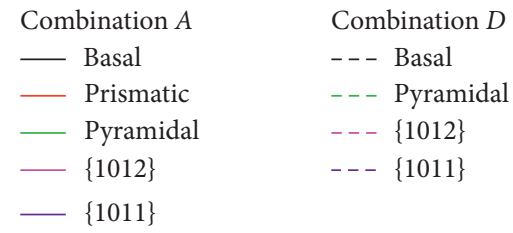

(b)
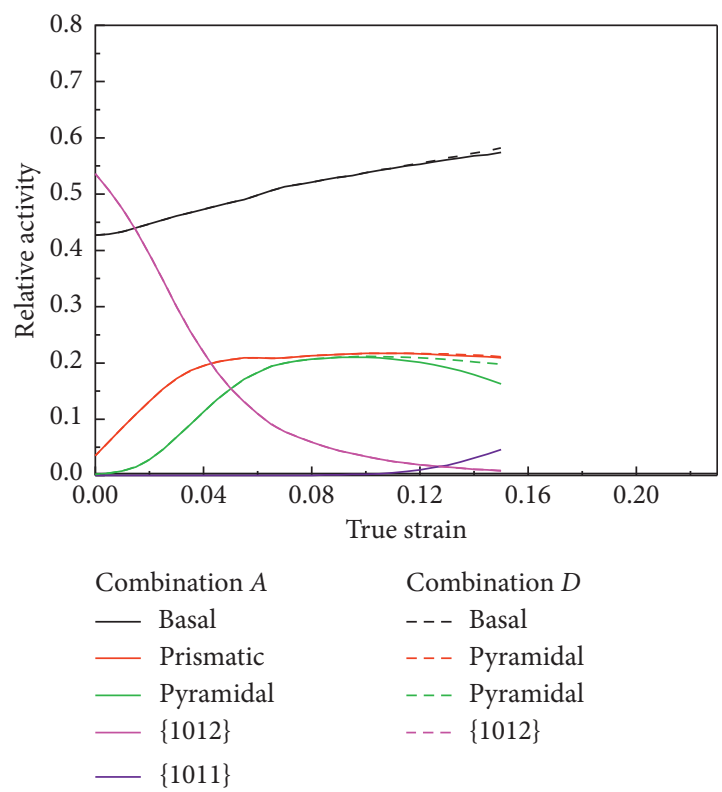

(d)

Figure 5: (a) Predicted stress-strain curves of AZ31 magnesium alloy under axial compression; (b)-(d) corresponding slip and twinning activities predicted by the VPSC model in the cases of combinations $\mathrm{B}, \mathrm{C}$, and D respectively.

deformation mechanism, the basal $\langle a\rangle$ slip, pyramidal $<c+a>$ slip, $\{10 \overline{1} 2\}$ tension twin, and $\{10 \overline{1} 1\}$ compression twin displayed increased activity. Moreover, the corresponding stress-strain curve was relatively increased, which is consistent with the important influence of the prismatic $<a>$ slip on ultimate strength during deformation, as observed by Kang et al. [21]. When the pyramidal $\langle c+a\rangle$ slip was removed, as shown in Figure 5(c), changes were noted with respect to the activities in the basal $\langle a\rangle$ slip, prismatic $<a>$ slip, and $\{10 \overline{1} 1\}$ compression twin, with evident changes in the $\{10 \overline{1} 1\}$ compression twin. When the $\{10 \overline{1} 1\}$ compression twins were not considered in the VPSC model, minimal influence was observed on the activities of other deformation mechanisms in the entire deformation process, as shown in Figure 5(d).

Figure 6 shows the predicted stress-strain curves in the axial tension process and corresponding slip and twinning activities in the different combinations of deformation mechanisms. As illustrated in Figure 6(a), the stress-strain curve of combination $A$ was nearly identical to that of combination $C$. On the contrary, the stress-strain curves of combinations $B$ and $D$ varied significantly with large flow 

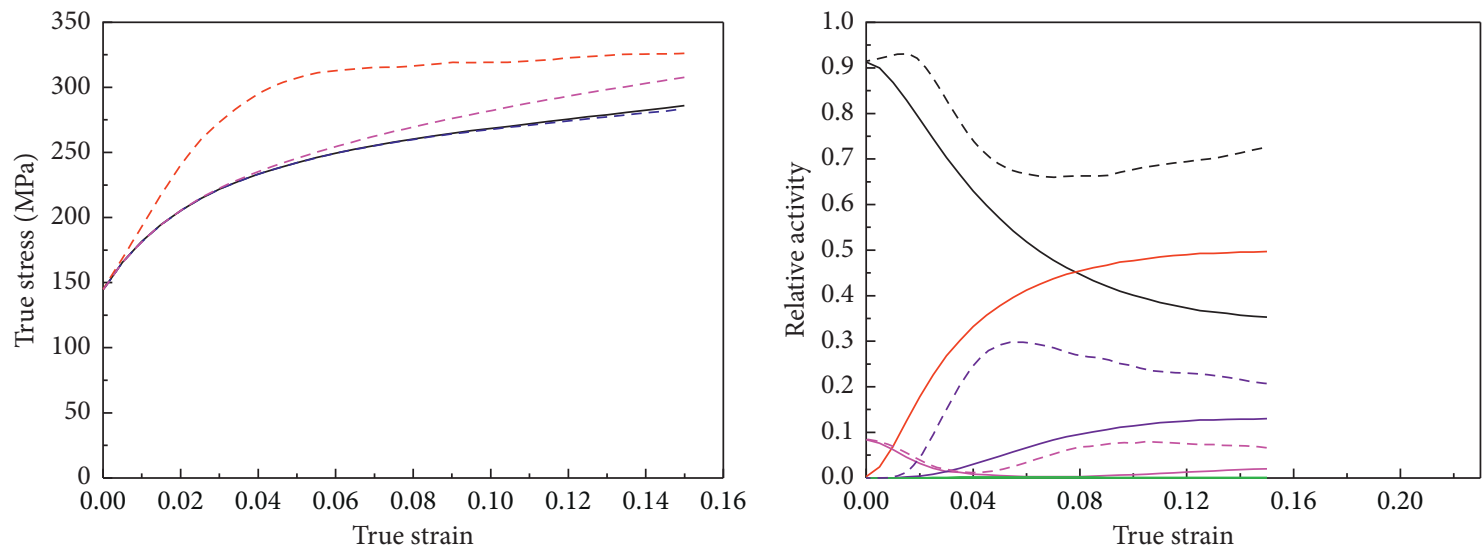

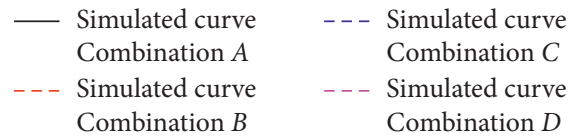

(a)

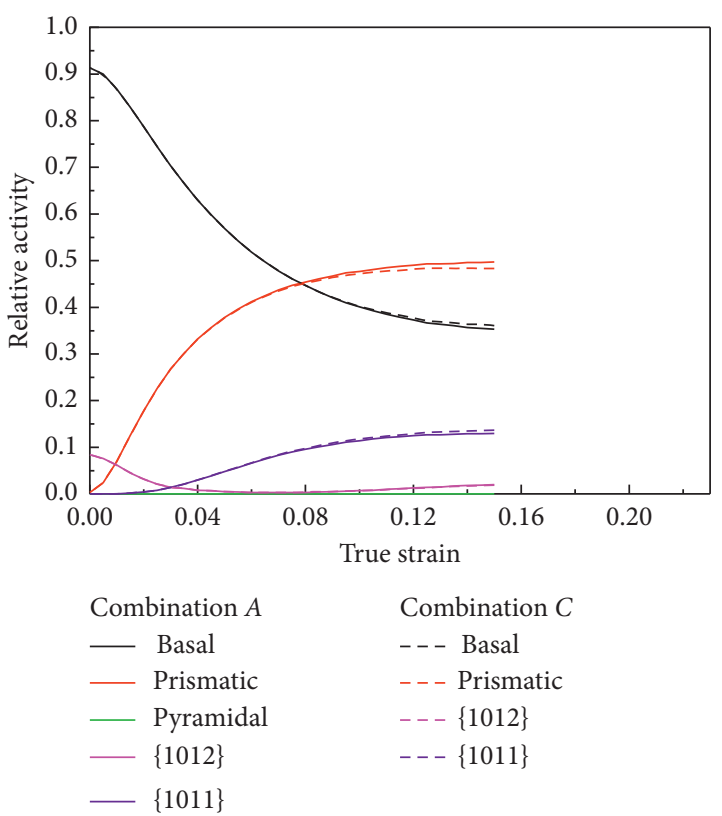

(c)

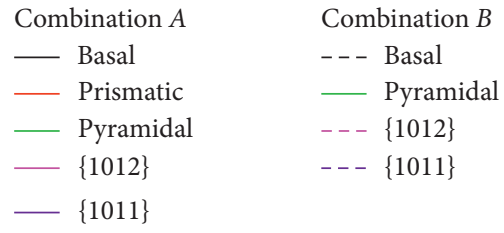

(b)

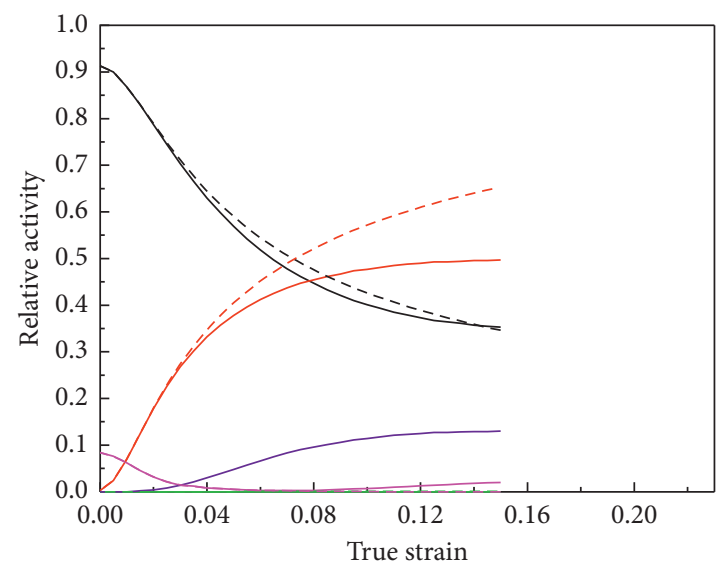

$\begin{array}{ll}\text { Combination } A & \text { Combination } D \\ - \text { Basal } & --- \text { Basal } \\ - \text { Prismatic } & --- \text { Pyramidal } \\ - \text { Pyramidal } & --- \text { Pyramidal } \\ -\{1012\} & ---\{1012\} \\ -\{1011\} & \end{array}$

(d)

FIgUre 6: (a) Predicted stress-strain curves of AZ31 magnesium alloy under axial compression; (b-d) corresponding slip and twinning activities predicted by the VPSC model in the cases of combinations $\mathrm{B}, \mathrm{C}$, and D respectively.

stress and yield limits. Figure 6(b) presents that the $c$-axis distribution of the grain was perpendicular to the ED during axial tension and the compressive stress was inconducive to the activity of the tension twins, such that the activity of the tension twin was small. When prismatic $\langle a\rangle$ slip was not considered in the VPSC model, the increment in the activity of the $\{10 \overline{1} 1\}$ compression twins was the highest in comparison with other deformation mechanisms at the initial stage. When the pyramidal $\langle c+a\rangle$ slip was not embedded into the VPSC model, Figure 6(c) illustrates that the activities in other deformation mechanisms remained unaffected whether it existed or not. Therefore, the slip has a negligible influence on the mechanical response of axial tension. When the $\{10 \overline{1} 1\}$ compression twins were not considered, as shown in Figure 6(d), the activity of prismatic $<a>$ slip changed significantly combined with its stressstrain curve. The study determined that the substantial increase is conducive for improving flow stress in the axial tensile process.

Slip and twinning activities have a direct influence on the texture evolution of AZ31 magnesium alloy during plastic deformation. Therefore, this study used the VPSC model 


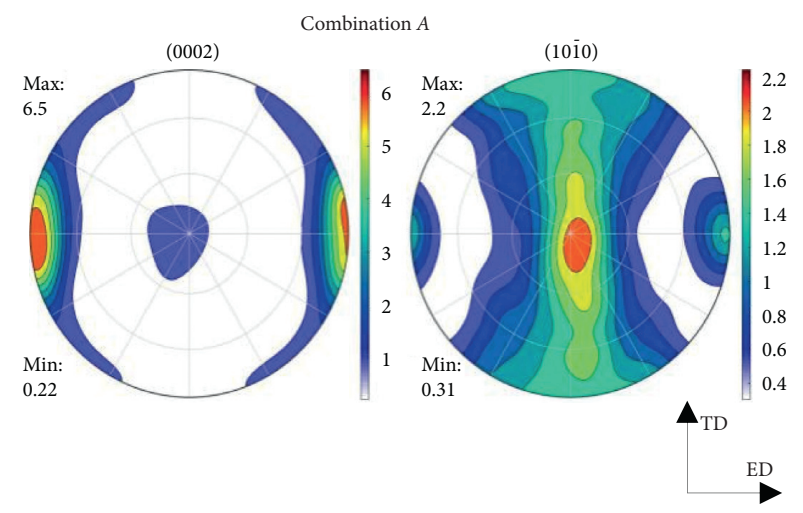

(a)

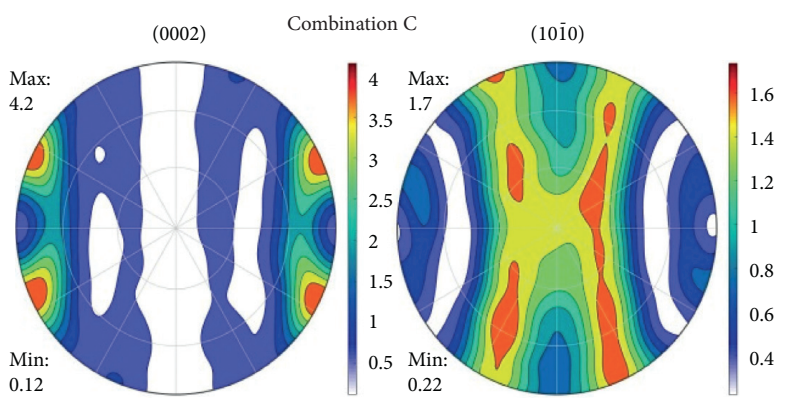

(c)

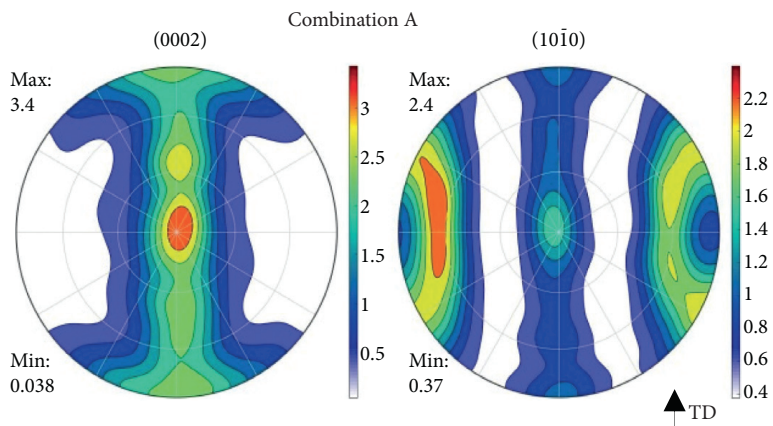

(e)

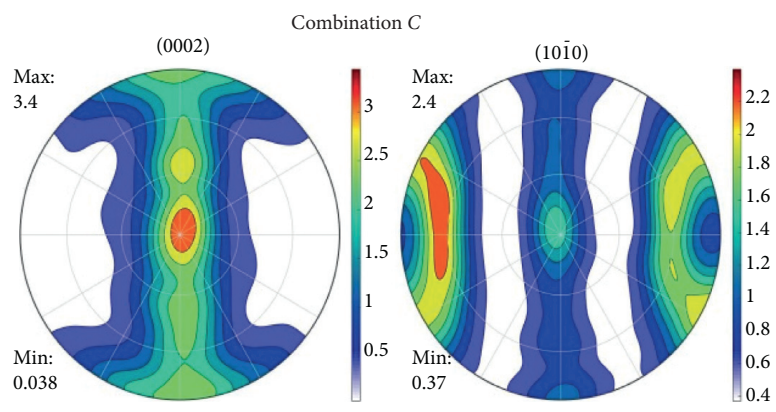

(g)

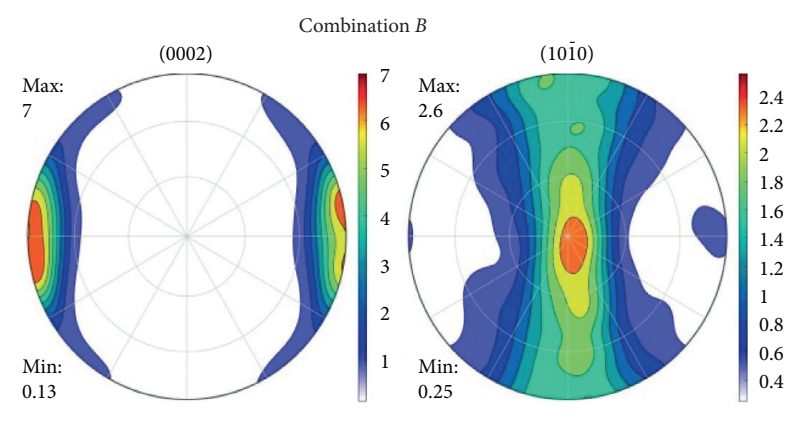

(b)

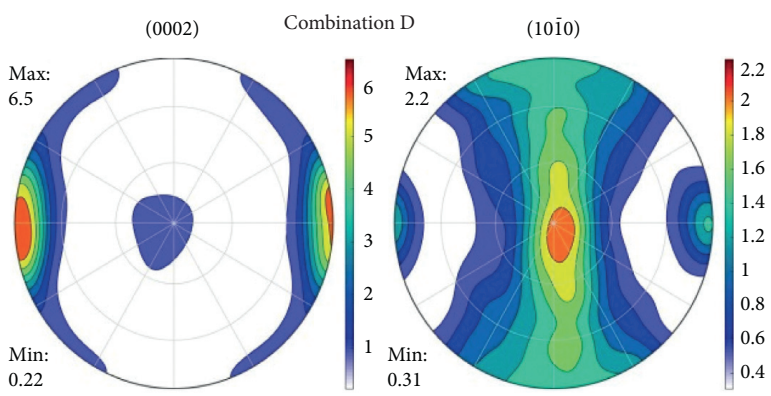

(d)

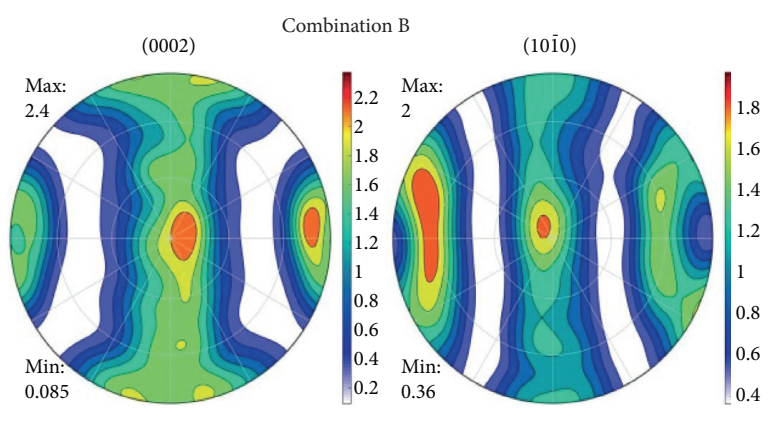

(f)

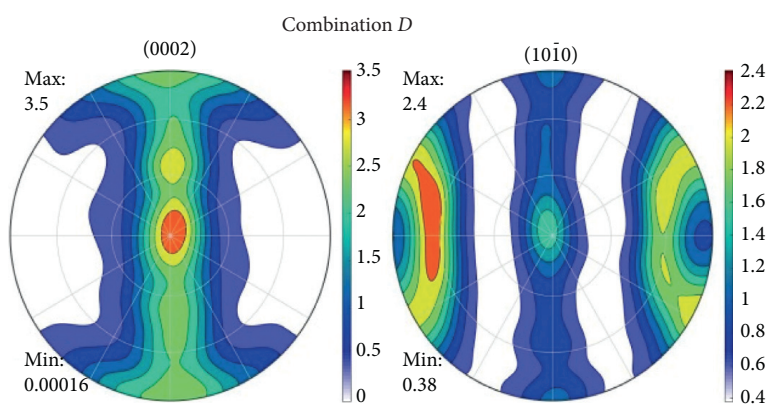

(h)

FIgURE 7: Predicted texture under (a-d) axial compression and (e-h) axial tension at a strain of 0.15 with different combinations of deformation mechanisms.

with different combinations of deformation mechanisms to predict texture evolution when the true strain in axial compression and tension was 0.15, as shown in Figure 7. During axial compression, the distribution of pole densities in the $\{0002\}$ and $\{1010\}$ pole figures of combinations $A$ and $D$ was nearly the same. However, in the case of combination $B$, the pole density of the $\{0002\}$ basal texture completely shifted to the ED. In the case of combination $C$, the splitting 

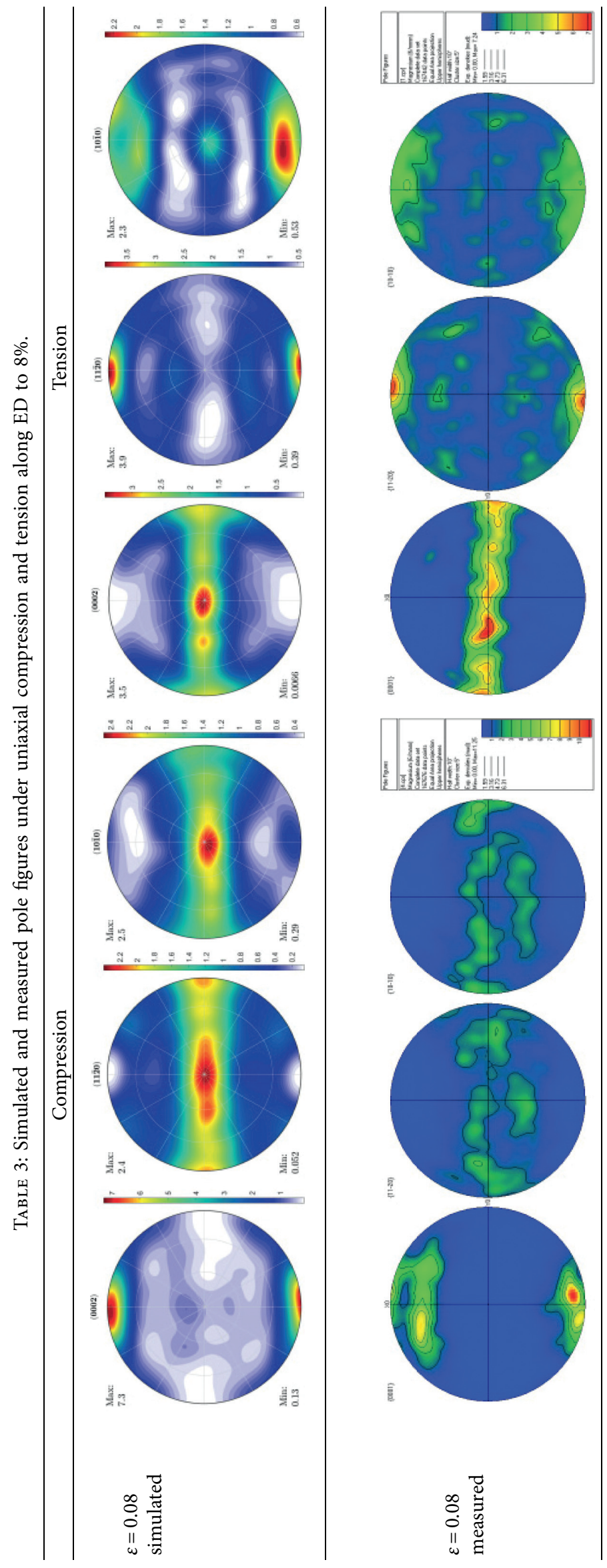
of pole densities was evident around the ED of $\{0002\}$, and several pole densities gradually deviated toward the ND. On the contrary, in the case of combinations $A, C$, and $D$ during axial tension, the $\{0002\}$ and $\{1010\}$ pole figures exhibited only minor changes, as shown in Figures $7(\mathrm{e})-7(\mathrm{~h})$, respectively. However, in the case of combination $B$, the ED of the $\{0002\}$ pole figure texture showed an evident pole density distribution.

The aforementioned differences with respect to predicted texture can also be related to the slip and twinning activities during plastic deformation. In the case of combination $B$, when uniaxial compression occurred along the ED with an increase in the activity of the pyramidal $\langle c+a\rangle$ slip, the pole density of the basal texture $\{0002\}$ completely shifted to the ED. However, when uniaxial tension occurred along the $\mathrm{ED}$, the activity increment of pyramidal $\langle c+a\rangle$ slip was very small, such that it had minimal influence on the prediction of texture. The distribution in the ED of the pole figure texture was caused by the massive activity of the $\{10 \overline{1} 1\}$ compression twins. In the case of combination $C$, when uniaxial compression occurred along the ED, an increase in the activity of the $\{10 \overline{1} 1\}$ compression twin led to a rotation of $\sim 56^{\circ}$ on the $c$-axis in the crystals, which contributed to the shift of pole density of the $\{0002\}$ pole figure texture toward the ND. In addition, combined with the prismatic $\langle a\rangle$ slip, it could generate a rotation around the $c$-axis in HCP metals found by Chapuis et al. [22]. The pole density division in the ED is generally caused by increased activity in the prismatic $\langle a\rangle$ slip. From combination $D$, we determined whether the $\{10 \overline{1} 1\}$ compression twins existed or not, which will not affect the texture prediction of the uniaxial tension and compression along the ED. Increased activity in the prismatic $<a>$ slip is helpful for improving the flow stress in the axial tension deformation process.

Table 3 shows the simulated and measured pole figures under uniaxial compression and tension along ED to $8 \%$ by using the VPSC model of combination $A$. Through comparison, it can be seen that the simulation results are in good agreement with the experimental results, which reasonably reflects the texture evolution of compression and tension along the ED direction, and verified the accuracy of the VPSC model in simulating the texture evolution of magnesium alloy in this study.

\section{Conclusion}

This study simulated and predicted the mechanical response and texture evolution of squeezed AZ31 magnesium alloy by using the VPSC model with different combinations of deformation mechanisms. The influence of secondary deformation mechanisms $A, B$, and $C$ on the plastic deformation of AZ31 magnesium alloy can be concluded as follows.

(1) The increment in the activity of prismatic $\langle a\rangle$ slip is conducive for improving flow stress during axial tension deformation and the splitting of pole density around the ED in the $\{0002\}$ pole figure during axial compression.

(2) The increment in the activity of pyramidal $\langle c+a\rangle$ contributes to the transfer of the basal texture to the
$\mathrm{ED}$ in the $\{0002\}$ pole figure during axial compression.

(3) The $\{10 \overline{1} 1\}$ compression twin has a negligible influence on the plastic deformation and mechanical response of AZ31 magnesium alloy. However, during axial compression, a large increase in activity is helpful for transferring the pole density to the ND in the $\{0002\}$ pole figure texture.

\section{Data Availability}

The data used to support the findings of this study are included within the article.

\section{Conflicts of Interest}

The authors declare that they have no conflicts of interest.

\section{References}

[1] X. Huang, K. Suzuki, and N. Saito, "Microstructure and mechanical properties of AZ80 magnesium alloy sheet processed by differential speed rolling," Materials Science \& Engineering, vol. 508, no. 1-2, pp. 226-233, 2009.

[2] D. L. Yin, J. T. Wang, J. Q. Liu et al., "On tension-compression yield asymmetry in an extruded $\mathrm{Mg}-3 \mathrm{Al}-1 \mathrm{Zn}$ alloy," Journal of Alloys and Compounds, vol. 478, no. 1-2, pp. 0-795, 2009.

[3] M. H. Yoo, "Slip, twinning, and fracture in hexagonal closepacked metals," Metallurgical and Materials Transactions, vol. 12, no. 3, pp. 409-418, 1981.

[4] A. Pandey, F. Kabirian, J. H. Hwang et al., "Mechanical responses and deformation mechanisms of an AZ31 Mg alloy sheet under dynamic and simple shear deformations," International Journal of Plasticity, vol. 68, pp. 111-131, 2015.

[5] S. R. Agnew, M. H. Yoo, and C. N. Tomé, "Application of texture simulation to understanding mechanical behavior of $\mathrm{Mg}$ and solid solution alloys containing $\mathrm{Li}$ or Y," Acta Materialia, vol. 49, no. 20, pp. 4277-4289, 2001.

[6] S. R. Agnew and O. Duygulu, "Plastic anisotropy and the role of non-basal slip in magnesium alloy AZ31B," International Journal of Plasticity, vol. 21, no. 6, pp. 1161-1193, 2005.

[7] R. A. Lebensohn and C. N. Tomé, "A self-consistent anisotropic approach for the simulation of plastic deformation and texture development of polycrystals: Application to zirconium alloys," Acta Metallurgica et Materialia, vol. 41, no. 9, pp. 2611-2624, 1993.

[8] R. J. Asaro and A. Needleman, "Overview no. 42 Texture development and strain hardening in rate dependent polycrystals," Acta Metallurgica, vol. 33, no. 6, pp. 923-953, 1985.

[9] F. Kabirian, A. S. Khan, and T. GnäUpel-Herlod, "Viscoplastic modeling of mechanical responses and texture evolution in extruded AZ31 magnesium alloy for various loading conditions," International Journal of Plasticity, vol. 68, pp. 1-20, 2015.

[10] R. A. Lebensohn, P. A. Turner, J. W. Signorelli et al., "Calculation of intergranular stresses based on a large-strain viscoplastic self-consistent polycrystal model," Modelling and Simulation in Materials Science and Engineering, vol. 6, no. 4, p. 447, 1999.

[11] C. N. Tomé, R. A. Lebensohn, and U. F. Kocks, “A model for texture development dominated by deformation twinning: application to zirconium alloys," Acta Metallurgica Et Materialia, vol. 39, no. 11, pp. 2667-2680, 1991. 
[12] A. Staroselsky and L. Anand, "A constitutive model for hcp materials deforming by slip and twinning: application to magnesium alloy AZ31B," International Journal of Plasticity, vol. 19, pp. 1843-1864, 2003.

[13] J. W. Hutchinson, "Bounds and self-consistent estimates for creep of polycrystalline materials," in Proceedings of the Royal Society of London. Series A, Mathematical and Physical Sciences, vol. 348, no. 1652, pp. 101-127, London, UK, Feburary 1976.

[14] R. Lebensohn and C. Tomé, Manual for Code Visco-Plastic Self-Consistent (Vpsc), Version $7 b$, Los Alamos National Laboratory, Los Alamos, NM, USA, 2007.

[15] X. Y. Lou, M. Li, R. K. Boger et al., "Hardening evolution of AZ31B Mg sheet," International Journal of Plasticity, vol. 23, no. 1, pp. 44-86, 2007.

[16] J. Koike, "Enhanced deformation mechanisms by anisotropic plasticity in polycrystalline $\mathrm{Mg}$ alloys at room temperature," Metallurgical and Materials Transactions A (Physical Metallurgy and, Materials Science), vol. 36, no. 7, pp. 1689-1696, 2005.

[17] R. E. Reed-Hill and W. D. Robertson, "Additional modes of deformation twinning in magnesium," Acta Metallurgica, vol. 5, no. 12, 1957.

[18] Q. Ma, H. E. Kadiri, A. L. Oppedal et al., "Twinning effects in a rod-textured AM30 Magnesium alloy," International Journal of Plasticity, vol. 29, pp. 60-76, 2012.

[19] S. R. Agnew, D. W. Brown, and C. N. Tomé, "Validating a polycrystal model for the elastoplastic response of magnesium alloy AZ31 using in situ neutron diffraction," Acta Materialia, vol. 54, no. 18, pp. 4841-4852, 2006.

[20] B. Hutchinson, M. R. Barnett, A. Ghaderi, P. Cizekb, and I. Sabirovbet, "Deformation modes and anisotropy in magnesium alloy AZ31," International Journal of Materials Research, vol. 100, no. 4, pp. 556-563, 2009.

[21] J. Y. Kang, B. Bacroix, and R. Brenner, "Evolution of microstructure and texture during planar simple shear of magnesium alloy," Scripta Materialia, vol. 66, no. 9, pp. 654-657, 2012.

[22] A. Chapuis, Z. Q. Wang, and Q. Liu, "Influence of material parameters on modeling plastic deformation of $\mathrm{Mg}$ alloys," Materials Science and Engineering: A, vol. 655, pp. 244-250, 2016. 